36. Pitchenik AE, Rubinson HA. The radiographic appearance of tuberculosis in patients with the acquired immunodeficiency syndrome (AIDS) and pre-AIDS. Am Rev Respir Dis 1985;131:393-396.

37. Havlir DV, Ellner JJ. Mycobacterium avium complex. In: Mandell GL, Bennett JE, Dolin R, eds. Principles and Practice of Infectious Diseases. New York, NY: Churchill Livingstone; 1995:2250-2264
38. Tenholder MF, Moser RJ, Tellis CJ. Mycobacteria other than tuberculosis: pulmonary involvement in patients with acquired immunodeficiency syndrome. Arch Intern Med 1988;148:953-955.

39. Ruf B, Schuermann D, Brehmer W. Pulmonary manifestations due to Mycobacterium avium-Mycobacterium intracellulare (MAI) in AIDS patients. Am Rev Respir Dis 1990:141(suppl):A611. Abstract.

\title{
First Reported Case of Occupationally Acquired HIV From Autopsy
}

\section{Gina Pugliese, RN, MS; Martin S. Favero, PhD}

Physicians at the Vanderbilt University School of Medicine recently reported a case of occupationally acquired HIV infection in a boardcertified pathologist who sustained a scalpel wound to the hand while performing an autopsy on a patient with AIDS and severe progressive multifocal leukoencephalopathy. ${ }^{1}$ The autopsy was begun at approximately $8 \mathrm{PM}-$ 5 hours post mortem and 12 hours into the pathologist's workday. Precautions for autopsies on patients with HIV, as outlined in the institution's guidelines, were observed strictly. The pathologist wore a disposable "spacesuit" with hood, waterproof apron, surgical mask with face shield, shoe covers, sleeve protectors, and two pairs of latex gloves. Except in very rare cases, coarse-mesh chain-mail gloves were not being used by staff, owing to their clumsiness, particularly when holding instruments, and to uncertainty about their efficacy in preventing stab wounds.

The scalpel wound occurred while reflecting the scalp from the head, which was stabilized with a standard head block. The pathologist's left hand slipped toward the scalpel resulting in a wound approximately $1 \mathrm{~cm}$ in depth in the left first digit. The wound immediately bled and filled the inner glove. Immediate removal of the glove was followed by extensive rinsing with a $5.25 \%$ solution of sodium hypochlorite (undiluted Clorox), a wash with water and povidone-iodine, and then a second wash with hypochlorite while the wound was retracted open. A decision was made not to institute AZT prophylaxis. HIV-1 antibody ELISA testing of the pathologist was negative at baseline (day 1 after injury) and weakly positive at 6 weeks (confirmed by Western blot P-24 positivity), indicating early seroconversion. Although definite autopsy-acquired HIV-1 infection has not been reported prior to this case, autopsy injuries are common, and autopsies are recognized as a potential route of HIV transmission. Studies have shown that injuries occur in approximately 1 of 11 autopsies performed by residents and in 1 of 55 performed by experienced pathologists. Moreover, the risk of transmission of HIV increases with exposure to blood from terminally ill patients, probably reflecting the higher titers of HIV late in the course of AIDS. This case documents that scalpel wounds sustained during autopsies of patients with AIDS can result in HIV1 infection. The authors note that another recent report documents the acquisition of HIV infection by a scalpel injury in a surgeon. ${ }^{2}$ Although the rate of seroconversion in the autopsy setting is unknown, the use of saws and scalpels during autopsy (and surgery), which may result in deep wounds, increase the risk of seroconversion after injury. The authors note that pathologists should be aware that $67 \%$ of scalpel wounds are inflicted in the "oppositional area consisting of the distal thumb, index and middle finger of the nondominant hand," which typically retracts during autopsy. Thus, protection of the retracting hands remains critical. The use of Kevlar gloves, or similar gloves that are cut-resistant, worn with two pairs of latex gloves is a practical alternative to the chain-mail gloves.

A recent study reported that viral HIV-1 titers decline over the 24 hours after death, resulting in no culturable HIV-1 after 21 hours. Delaying autopsies therefore has been suggested as a simple means of risk reduction. However, others have isolated HIV-1 from brain 24 hours postmortem and from bone, spleen, brain, and blood up to 6 days postmortem, underscoring the persistence of risk even in delayed cases. Thus, postmortem viral burden is variable and influenced by numerous factors, including viral burden at death, viral strain, premortem antiviral therapy, and morgue temperature.

FROM: 1. Johnson MD, Schaffner W, Atkinson J, Pierce MA. Autopsy risk and acquisition of HIV infection. Arch Pathol Lab Med 1997;121:64-66.

2. Ippolito $G$, Italian study for occupational risk of HIV. Scalpel injury and HIV infection in a surgeon. Lancet 1996;347:1042. 